\title{
Multi-Target Detection and Tracking Using Dynamic Programming
}

$$
\text { Jingmei Li }{ }^{1, a} \text {, Chonglei Liü,b }
$$

${ }^{1}$ Science and Technology on Underwater Acoustic Antagonizing Laboratory, Beijing 100036, China

\author{
${ }^{2}$ Institute of Acoustics, CAS, Beijing 100190, China \\ alijingmei6072@gmail.com, ${ }^{\mathrm{b}}$ liucl@ioa.cn
}

Keywords: Multi-target detection, tracing, Dynamic programming

\begin{abstract}
A dynamic programming (DP) multi-target detection and tracking algorithm is developed for the simultaneous detection and tracking of low signal - noise ratio (SNR) targets in passive sonar processing. The detecting and tracking approach is considered as a special problem of multiple dimensional image processing and integrated into a single optimization procedure. The DP algorithm is extended to passive sonar processing and a new trace-evaluation function is proposed in this paper. Numerical simulation result shows the advantage of DP algorithm.
\end{abstract}

\section{Introduction}

The Dynamic Programming (DP) algorithm was developed by Bellman [1] to solve multistage optimal control problems and found its uses in optimal decision and target tracking search fields. The DP technique has been proved effective in many applications such as computer vision and IR camera. Instead of applying any threshold process normally used by conventional algorithm, the DP approach take into account all the raw target information. By searching all the possible target state sequences in a multi-dimensional data space to detect target paths, the DP technique shows its advantages of higher sensitivity to weak targets and robust performance to interference and vehicle maneuvers. Another distinguishing feature of DP approach is that targets detection and their tracking are performed in a single optimization procedure and the paths are scored by summing the values of a statistical cost function, which is separated into a set of independent scalar functions.

The signals coming from the targets and ocean environment are processed to get time / bearing history in passive sonar's post-processing and the display of this history is used to detect and track targets. To be treated as a specific two-dimensional image, the time / bearing history is separated into a succession of discrete stages and is able to make transitions from one stage to another through optimal decision. The possible target paths are detected and keep tracking by the assigned value through a cost function or trace-evaluation function. The design of the cost function is based on the ratio of posteriori probabilities, which uses the observation, and above stages' transition law.

\section{Target motion and measurement model}

Provided that the target motion is approximately linear in bearing space and the state innovations can be modeled as a first order Markov random walk. The target state vector for kth state is defined as

$$
\mathrm{z}(\mathrm{k})=(\mathrm{x}(\mathrm{k}), \mathrm{vx}(\mathrm{k}), \mathrm{y}(\mathrm{k}), \mathrm{vy}(\mathrm{k}))
$$

The target state update equation is:

$$
\begin{aligned}
& x(k+1)=x(k)+v x(k) * T+a x(k) * T^{*} T+e x(k) . \\
& y(k+1)=y(k)+v y(k) * T+a y(k) * T^{*} T+e y(k) . \\
& v x(k+1)=v x(k)+a x(k) * T . \\
& v y(k+1)=v y(k)+a y(k) * T .
\end{aligned}
$$


Where $\operatorname{vx}(\mathrm{k}) \mathrm{xy}(\mathrm{k})$ and $\mathrm{ax}(\mathrm{k}) \mathrm{ay}(\mathrm{k})$ is the target moving rate and acceleration, ex(k) ey(k) is a zero- mean random processes, $\mathrm{T}$ is the measure period.

The observations that provided to the DP module are the amplitude output of sonar preprocessor. The amplitude measurement is:

$$
r(\mathrm{k})=\left\{\begin{array}{c}
S(\mathrm{k})+\mathrm{w}(\mathrm{k}) \\
W(\mathrm{k}) .
\end{array}\right.
$$

where $\mathrm{s}(\mathrm{k})$ is target amplitude and $\mathrm{w}(\mathrm{k})$ is additive noise with known statistics.

\section{DP approach}

The general DP solution of a target tracking problem is to select a sequence of track states (x(n), $\mathrm{x}(\mathrm{n}-1), \ldots, \mathrm{x}(1))$ that maximize a scoring function $\mathrm{g}$ reflecting models for target amplitude and motion behavior [2]. For a first order Markov random walk model, the scoring function could be separated into the sum of functions:

$g(x(n), x(n-1), \ldots, x(1))=g(n)(x(n), x(n-1))+g(n-1)(x(n-1), x(n-2))+\ldots+g(1)(x(2), x(1))$

The DP stage maximize procedure is separated as

$$
\begin{aligned}
& \mathrm{K}(1)=\max [\mathrm{g}(1)(\mathrm{x}(2), \mathrm{x}(1))] \\
& \mathrm{K}(\mathrm{n}-1)=\max [\mathrm{K}(\mathrm{n}-2)+\mathrm{g}(\mathrm{n}-1)(\mathrm{x}(\mathrm{n}-1), \mathrm{x}(\mathrm{n}-2))] .
\end{aligned}
$$

The scoring function $g$ is defined as the logarithm of the ratio of posteriori probabilities:

$$
\mathrm{g}(\mathrm{x}(\mathrm{n}), \mathrm{x}(\mathrm{n}-1), \ldots, \mathrm{x}(1))=\log [\mathrm{P}(\mathrm{z}(\mathrm{n}), \mathrm{z}(\mathrm{n}-1), \ldots, \mathrm{z}(1) \mid \mathrm{r}(\mathrm{n})) / \mathrm{P}(\mathrm{H} 0 \mid \mathrm{r}(\mathrm{n}))] \text {. }
$$

Where $(z(n), z(n-1), \ldots, z(1))$ is the hypothesis of the target state sequence at $n, H 0$ is non target hypothesis, and $r(n)$ is the measurements set. For a first order random model, the DP update equation is:

$$
\mathrm{K}(\mathrm{n}-1)=\log [\mathrm{p}(\mathrm{r}(\mathrm{n}) \mid \mathrm{z}(\mathrm{n})) / \mathrm{p}(\mathrm{r}(\mathrm{n}) \mid \mathrm{H} 0)]+\max [\log \mathrm{P}(\mathrm{z}(\mathrm{n}) \mid \mathrm{z}(\mathrm{n}-1))+\mathrm{K}(\mathrm{n}-2)] .
$$

Where $\mathrm{p}(\mathrm{r}(\mathrm{n}) \mid \mathrm{z}(\mathrm{n}))$ and $\mathrm{p}(\mathrm{r}(\mathrm{n}) \mid \mathrm{H} 0)$ are the probability density functions.

\section{Sonar application}

In passive sonar, the likelihood ratio is:

$$
\mathrm{p}(\mathrm{r}(\mathrm{n}) \mid \mathrm{z}(\mathrm{n})) / \mathrm{p}(\mathrm{r}(\mathrm{n}) \mid \mathrm{H} 0)=\mathrm{ps}(\mathrm{r}(\mathrm{n})-\mathrm{s}) / \mathrm{pw}(\mathrm{r}(\mathrm{n})) \text {. }
$$

Where ps and pw are the target add noise and noise only amplitude probability density functions, and $\mathrm{s}$ is the estimated value of amplitude. Under the Gaussian noise model suppose, the likelihood ratio is linear [3].

For a first order Gauss- Markov random walk model,

$$
\log \mathrm{P}(\mathrm{z}(\mathrm{n}) \mid \mathrm{z}(\mathrm{n}-1))=\log [\mathrm{P}(\mathrm{d}(\mathrm{x})) \mathrm{P}(\mathrm{d}(\mathrm{y})) \mathrm{P}(\mathrm{d}(\mathrm{vx})) \mathrm{P}(\mathrm{d}(\mathrm{vy}))]
$$

Where $\{d(x), d(y), d(v x), d(v y)\}$ is the state innovation. Both dimensions of the state 
innovation are independent Gaussian random variable, so

$$
\begin{aligned}
& \mathrm{P}(\mathrm{d}(\mathrm{x}))=\operatorname{erf}[(0.5+[\mathrm{d}(\mathrm{x}) / \mathrm{x}]) / \sigma \mathrm{x}]-\operatorname{erf}[([\mathrm{d}(\mathrm{x}) / \mathrm{x}]) / \sigma \mathrm{x}]-0.5) / \sigma \mathrm{x}] \\
& \mathrm{P}(\mathrm{d}(\mathrm{y}))=\operatorname{erf}[(0.5+[\mathrm{d}(\mathrm{y}) / \mathrm{y}]) / \sigma \mathrm{y}]-\operatorname{erf}[([\mathrm{d}(\mathrm{y}) / \mathrm{y}]) / \sigma \mathrm{y}]-0.5) / \sigma \mathrm{y}] \\
& \mathrm{P}(\mathrm{d}(\mathrm{vx}))=\operatorname{erf}[(0.5+[\mathrm{d}(\mathrm{vx}) / \mathrm{vx}]) / \sigma \mathrm{vx}]-\operatorname{erf}[([\mathrm{d}(\mathrm{vx}) / \mathrm{vx}]) / \sigma \mathrm{vx}]-0.5) / \sigma \mathrm{vx}] \\
& \mathrm{P}(\mathrm{d}(\mathrm{vy}))=\operatorname{erf}[(0.5+[\mathrm{d}(\mathrm{vy}) / \mathrm{vy}]) / \sigma \mathrm{vy}]-\operatorname{erf}[([\mathrm{d}(\mathrm{vy}) / \mathrm{vy}]) / \sigma \mathrm{vy}]-0.5) / \sigma \mathrm{vy}] .
\end{aligned}
$$

Where erf is the standard Gaussian error function, $\sigma \mathrm{x} \sigma \mathrm{y}$ and $\sigma \mathrm{vx} \sigma \mathrm{vy}$ are the standard deviation of the target innovations.

\section{Results of simulation}

Some simulation based on DP algorithm is presented in this paper. The sonar has a uniform circular array. The raw data in the bearing / time display is a 128*256 matrix and there are four different SN targets' paths in it. Fig.1 shows the original display, and fig.2 is the processing result of the raw data.

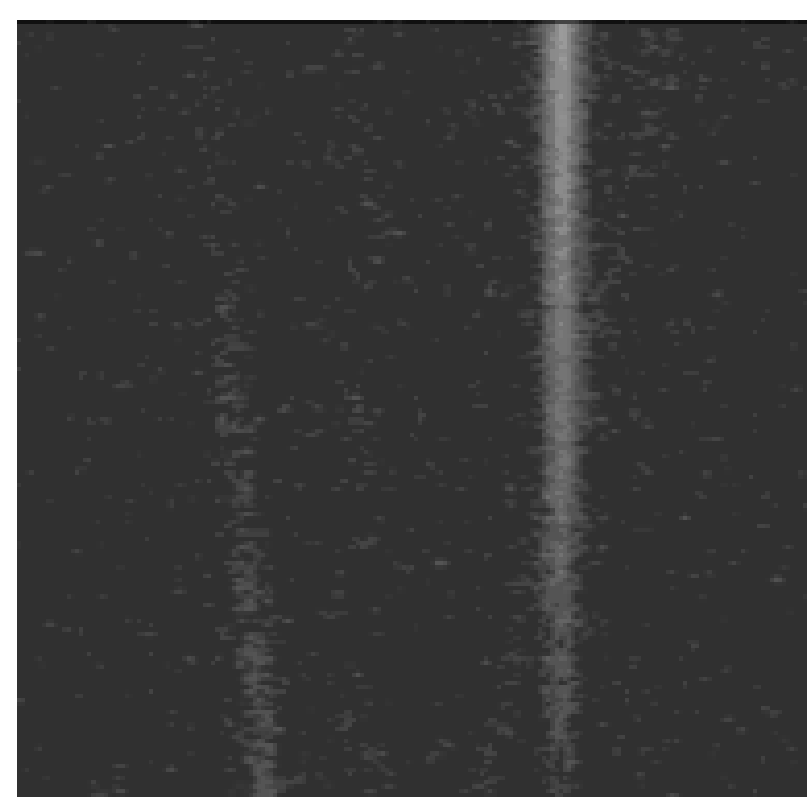

Fig.1 Original display

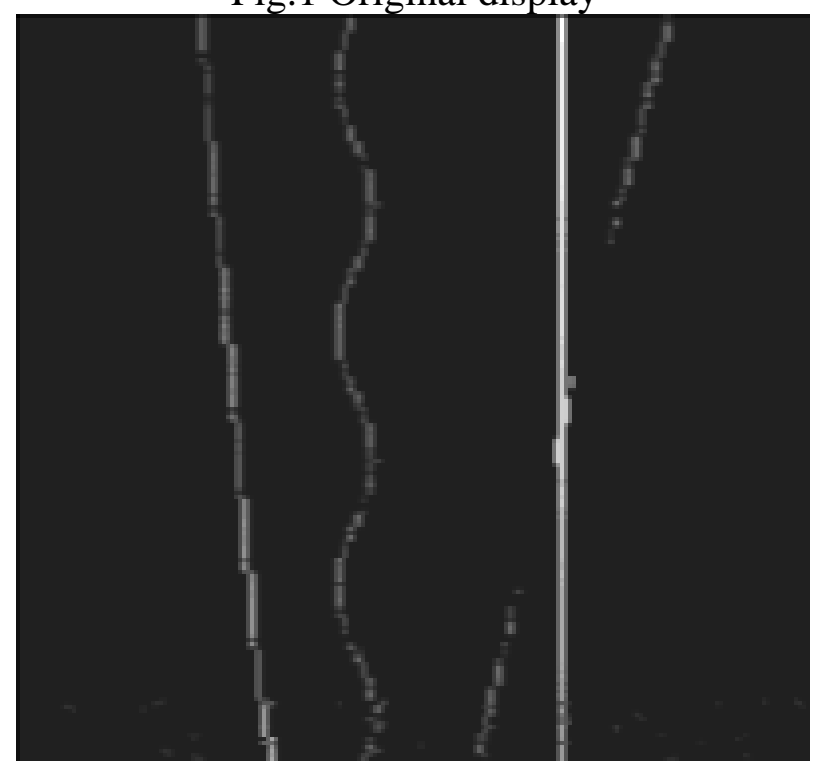

Fig.2 Results using DP algorithm 


\section{References}

[1] Bellman, Richard: Dynamic Programming. Princeton, NJ: Princeton University Press, 1957.

[2] Arnold, J., and Shaw, S. Efficient Target Tracking Using Dynamic Programming. IEEE Trans. on AES, AES-29 (1993), p44-55.

[3] Li, Q: Introduction on Sonar Processing. Beijing: Ocean Press, 1985.

[4] Barniv.Y: Dynamic Programming Solution for Detecting Dim Moving Targets. IEEE Trans. on AES, AES-21 (1985), p144-155.

[5] Information on http://en.wikipedia.org/wiki/Dynamic_programming\#cite_ref-4

[6] D. P. Bertsekas,Dynamic Programming and Optimal Control (VolumeOne). Belmont, MA: Athena Scientific, 1995

[7] E. A. Feinberg and A. Schwartz, "Constrained dynamic programmingwith two discount factors: applications and an algorithm,” IEEE Trans.Automat. Contr., vol. 44, pp. 628-631, Mar. 1999.

[8] D. H. Jacobson, "Optimal stochastic linear systems with exponentialperformance criteria and their relation to deterministic differentialgames,”IEEE Trans. Automat. Contr., vol. AC-18, pp. 124-131, Jan.1973

[9] H. L. Van Trees, Detection, Estimation and Modulation Theory, NewYork: Wiley, 1968 